\title{
Construction of sized eukaryotic cDNA libraries using low input of total environmental metatranscriptomic RNA
}

\author{
Rajiv Kumar Yadav ${ }^{1 *}$, Florian Barbi ${ }^{1}$, Antoine Ziller ${ }^{1}$, Patricia Luis ${ }^{1}$, Roland Marmeisse ${ }^{1}$, M Sudhakara Reddy ${ }^{2}$
} and Laurence Fraissinet-Tachet ${ }^{1}$

\begin{abstract}
Background: Construction of high quality CDNA libraries from the usually low amounts of eukaryotic mRNA extracted from environmental samples is essential in functional metatranscriptomics for the selection of functional, full-length genes encoding proteins of interest. Many of the inserts in libraries constructed by standard methods are represented by truncated cDNAs due to premature stoppage of reverse transcriptase activity and preferential cloning of short cDNAs.

Results: We report here a simple and cost effective technique for preparation of sized eukaryotic cDNA libraries from as low as three microgram of total soil RNA dominated by ribosomal and bacterial RNA. CDNAs synthesized by a template switching approach were size-fractionated by two dimensional agarose gel electrophoresis prior to PCR amplification and cloning. Effective size selection was demonstrated by PCR amplification of conserved gene families specific of each size class. Libraries of more than one million independent inserts whose sizes ranged between one and four kb were thus produced. Up to $80 \%$ of the insert sequences were homologous to eukaryotic gene sequences present in public databases.

Conclusions: A simple and cost effective technique has been developed to construct sized eukaryotic cDNA libraries from environmental samples. This technique will facilitate expression cloning of environmental eukaryotic genes and contribute to a better understanding of basic biological and/or ecological processes carried out by eukaryotic microbial communities.
\end{abstract}

Keywords: Metatranscriptomics, cDNA, cDNA library, mRNA, Gel electrophoresis

\section{Background}

The numerous eukaryotic microorganisms present in the environment potentially represent a rich source of genes encoding for novel enzymes or other proteins of interest in biotechnology. In this respect, functional metatranscriptomics has been demonstrated as a powerful tool in discovery of these genes [1-6]. Functional metatranscriptomics first requires the extraction of total RNA from environmental samples. Eukaryotic 3' polyadenylated (poly-A) messenger RNAs can then be purified from total RNA to remove the ribosomal RNA, other non-coding RNAs as well as the bacterial mRNAs that largely dominate

\footnotetext{
* Correspondence: rajiv-kumar.yadav@univ-lyon1.fr

'Ecologie Microbienne, UMR CNRS 5557, USC INRA 1364, Université Lyon 1, Université de Lyon, Villeurbanne, France

Full list of author information is available at the end of the article
}

environmental metatranscriptomes $[1,4,5]$. Poly-A mRNAs are then converted into cDNAs which are cloned in an appropriate expression vector. Such eukaryotic-specific environmental cDNA libraries, first described by Grant et al. [7], thus encompass protein coding genes expressed by the different eukaryotic microorganisms present in the original environmental sample [1,7]. Genes of interest can then be screened by expressing them in an appropriate eukaryotic system such as the yeast Saccharomyces cerevisiae [1,3-5].

Expression cloning of eukaryotic genes using reverse transcribed poly-A mRNA is a fundamental technology in molecular biology. However, obtaining libraries enriched in long cDNAs remains challenging for the production of functional proteins. The first step is the reverse transcription of mRNAs into cDNAs. This step is adversely affected by 
many factors and as a consequence, a large proportion of long mRNAs (e.g. larger than $1 \mathrm{~kb}$ ) is represented by $5^{\prime}$ truncated cDNAs [8]. Being small in size, these truncated cDNAs are preferentially amplified and cloned. Furthermore, functional metatranscriptomics most often involves the use of very low quantities of environmental eukaryotic mRNAs. This necessitates a highly efficient cDNA cloning approach which can make long eukaryotic transcripts available for functional studies.

Various library construction methods that enrich long cDNAs have been proposed. For example, several approaches that use the $5^{\prime}$ end-specific cap structure of eukaryotic poly-A mRNAs have been devised [9-14]. All these approaches however require many enzymatic and purification steps and as a consequence, relatively large quantities of starting poly-A mRNAs are needed which, once again, are difficult to obtain from environmental samples where eukaryotic mRNAs are diluted among predominant bacterial RNAs. cDNA size fractionation by agarose gel electrophoresis is an alternative strategy which requires few enzymatic steps and allows the preparation of different sized cDNA libraries from a single RNA sample. Libraries enriched in long cDNAs were for example constructed by agarose gel mediated size fractionation of cDNA synthesized from mouse embryo and human brain $[15,16]$. However, despite the application of all these strategies, all of them also require microgram amounts of poly-A mRNAs and numerous clones in these cDNA libraries still represent truncated transcripts.

Reverse transcriptase (RT) template switching is another approach for generation of cDNAs resulting from the reverse transcription of entire RNAs [17]. This technique, implemented in the commercial SMART (Clontech) or Mint (Evrogen, Moscow, Russia) kits, makes use of two activities of MMLV RT. The first one is to add a few deoxycytidines $(\mathrm{dC})$ at the end of single strand (ss) cDNAs. The second one is to switch template and to reverse transcribe an oligonucleotide whose 3' deoxyguanosinerich sequence anneals to the $\mathrm{dC}$ stretch artificially added at the end of the ss cDNA. As a consequence all cDNAs are bordered at their $3^{\prime}$ end by the same oligonucleotide sequence which can be used for their amplification by PCR in combination with a primer sequence added to the poly-dT primer used to initiate reverse transcription the mRNA.

In the present investigation, we demonstrate an efficient method of construction of sized eukaryotic cDNA libraries from environmental samples such as soil. This method is adapted from Wellenreuther et al. [18] who combined template switching in combination with agarose gel mediated size fractionation prior to full cDNA PCR amplification to isolate long full-length genes using microgram amounts of purified human poly-A mRNA. In this study, we modified this method to accommodate small limiting amounts of total environmental RNA.

\section{Results and discussion}

\section{Eukaryotic cDNA synthesis from total soil RNA}

We extracted total RNA from three soil samples coming from contrasted geographic localities (Additional file 1). The presence of sharp bands in the Bioanalyser electrophoregram, corresponding to small and large subunits rRNA and of a wide mRNA smear (approx. from $0.2 \mathrm{~kb}$ to $5 \mathrm{~kb}$ ) suggested that these RNA samples were not degraded (data not shown). Extraction yields ranged from 330 to $980 \mathrm{ng}^{-g^{-1}}$ of soil and at least $3 \mu \mathrm{g}$ of total soil RNA were obtained from each sample.

According to Urich et al. [19], soil RNA can be constituted of up to $90 \%$ of non-coding sequences (essentially rRNA) and approximately only $7 \%$ of the coding sequences can originate from eukaryotes. Although these figures certainly differ from one soil to another, bacterial biomass seems to always dominate in soil [20]. As a consequence, purification of $\mu \mathrm{g}$ amounts of poly-A mRNAs, as recommended in most cDNA library construction protocols, can hardly be met. We therefore developed a protocol which makes use of (i) few $\mu$ g of total RNA as starting material and (ii) includes long range PCR amplification for the synthesis of long cDNAs. This protocol, implemented in the Mint-2 kit (Evrogen) allowed us to obtain ds cDNAs of eukaryotic origin from as low as 3 $\mu g$ of total soil RNA. Such a quantity may contain only a few ng of poly-A mRNA. Success of cDNA synthesis was demonstrated by PCR amplification of an EF1 $\alpha$ gene fragment (data not shown).

\section{Size fractionation and PCR amplification of eukaryotic cDNAs}

Getting a high number of clones having long inserts (e.g. above $1 \mathrm{~kb}$ ) is one of the main challenges in cDNA library construction. As suggested in [18], we performed size fractionation of cDNA prior to their amplification to facilitate both the synthesis of long cDNA and minimize the preferential amplification of short ones from complex mixtures of long and short cDNAs. In the current protocol, ds cDNAs were first amplified through three PCR cycles, to slightly increase cDNA quantities without affecting significantly their average length. Pre-amplified cDNAs were then separated by bi-dimensional agarose gel electrophoresis (Figure 1) to maximize complete separation of cDNAs and minimize cross-contamination of size fractions. The cDNA containing gel was left unstained to prevent the adverse effect of UV irradiation but also because of the low quantity of loaded cDNAs which prevented their visualization. Three different size fractions (A, 0.1-0.5 kb; B, 0.5-1 kb and C, 1-4 kb) were recovered and amplified by PCR using between 22 and 30 cycles. 


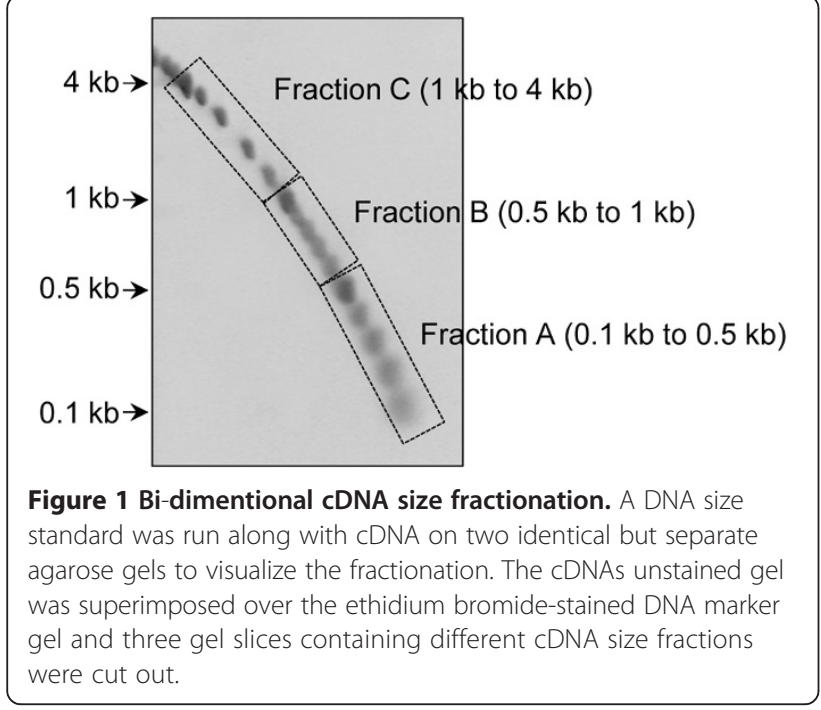

While amplified cDNAs without size fractionation gave a broad smear throughout the gel lane (Figure 2A), three distinct and discrete DNA smears (broad bands) corresponding to the different cDNA fractions were clearly visible (Figure 2B and Additional file 2A \& B). Fractions were tested for the absence of cross contaminations by PCR amplification of four eukaryotic genes of different length classes. For the 1-4 kb class, the conserved genes encode $\beta$-tubulin ( $\beta$-Tub) and EF1- $\alpha$ whose coding sequences are of about 1300 and 1400 bp respectively, while for the $0.5-1 \mathrm{~kb}$ class, they encode $40 \mathrm{~S}$ ribosomal protein S3 (RibS3) and peptide methionine sulphoxide reductase (MsrA) whose coding sequences are of about 800 and 600 bp respectively. As expected, positive amplifications of $\beta$-Tub and EF1- $\alpha$ fragments were only obtained for fractions $C(1-4 \mathrm{~kb})$, while
RibS3 and MsrA fragments were only detected for the intermediate $B$ fractions $(0.5-1 \mathrm{~kb})$ and no amplification was visible for the lowest A fractions (0.1-0.5 kb) (Figure 3). These results demonstrate that the method can not only be used for obtaining long cDNAs but also in getting high quality eukaryotic cDNA size fractions from very low amounts of starting total RNAs.

\section{Construction of sized cDNA libraries}

The three cDNA fractions from soil sample PL and the largest fraction $\mathrm{C}$ from sample $\mathrm{BB}$ were used for construction of cDNA libraries by directional cloning in the pFL61 yeast expression vector. All four libraries contained at least $10^{6}$ independent clones (range from $1.110^{6}$ for library PL-B to $2.610^{6}$ for library BB-C), but many more could have been obtained. After SfiI digestion of the library plasmid pools and their separation by agarose gel electrophoresis, the released cDNA insert pools of each library were detected as a smear whose size range corresponded to the original cDNA fraction size (Figure $2 \mathrm{C}$ and Additional file $2 \mathrm{C}$ ). The cDNA inserts of 42 random colonies from each of the three libraries from PL soil and 30 from library BB-C were PCR amplified. Absence of inserts was found for less than $1 \%$ of the plasmids and all of the PCR products fell within their expected size range (Additional file 3). For the $3 \mathrm{PL}$ libraries, ten inserts per library were sequenced from their 5 '-ends. Globally, $60 \%$ of the sequences returned a positive result upon BlastX searches against the Eukaryotic GenBank protein database $(80 \%, 70 \%$ and $30 \%$ for inserts from libraries PL-C, PL-B and PL-A respectively; Additional file 4).

\section{Conclusions}

We established a robust protocol to generate high quality eukaryotic sized cDNA libraries from total RNA extracted

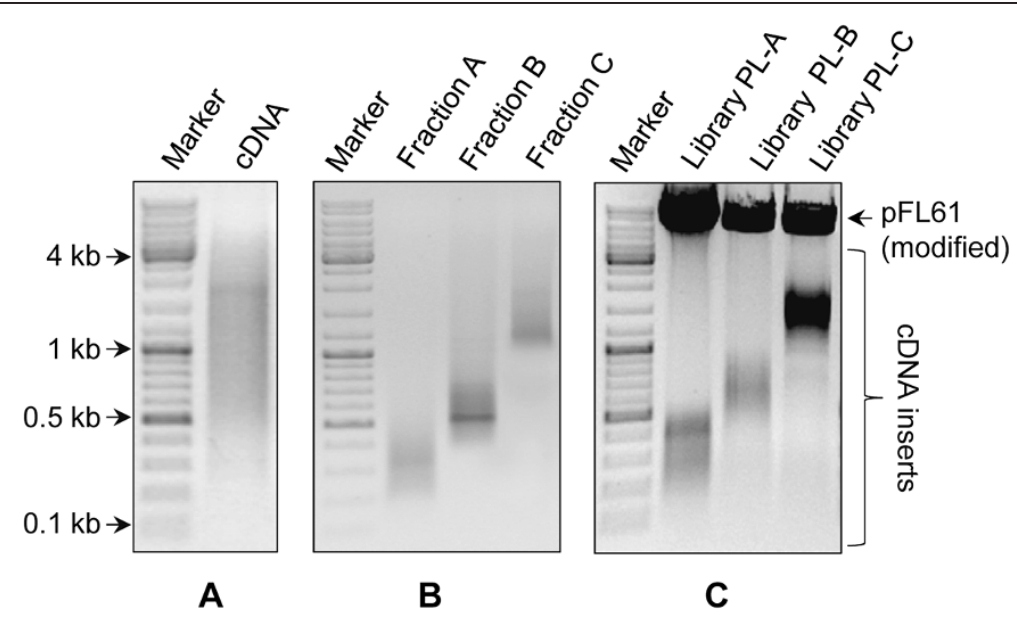

Figure 2 CDNA size fractions and libraries preparation from soil PL. Agarose gel separation of (A) amplified cDNA without size fractionation, (B) the three different CDNA fractions A (0.1-0.5 kb); B (0.5-1 kb) and C (1-4 kb) and (C) Sfil digested libraries PL-A, PL-B and PL-C. For each sized library, the size range of the cDNA inserts is similar before (B) and after (C) cloning. 


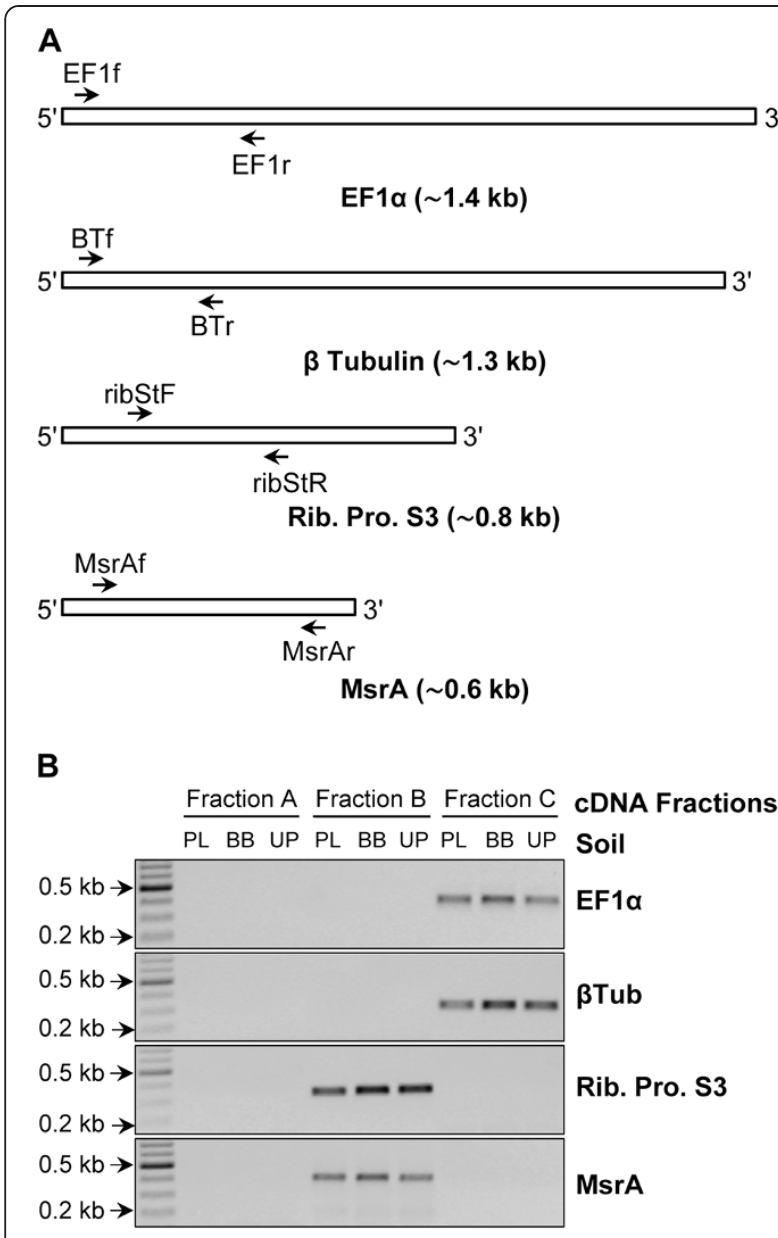

Figure 3 PCR validations of each CDNA size fraction obtained from soil samples PL, BB and UP. All three CDNA size fractions from each soil were tested for cross-contaminations by PCR amplification of conserved genes of different length. (A) Approximate size of coding sequences of each of the four tested genes and positions of the PCR primers. (B) Gel electrophoresis of the PCR products showing perfect correlation between sized CDNA fraction and detection of a PCR product from genes whose expected size is included in the corresponding fraction size range. In fraction C, only, elongation factor 1-alpha and beta-tubulin genes were amplified while in fraction $\mathrm{B}$, only ribosomal protein $\mathrm{S} 3$ and peptide methionine sulphoxide reductase genes were detected whereas none of theses genes was amplified from fraction $\mathrm{A}$.

from environmental samples. We demonstrated that the original protocol developed by Wellenreuther et al. [18] using micrograms amounts of purified poly-A mRNA extracted from human tissues can be considerably scaled down and implemented on total RNA from environments dominated by bacterial RNA. This protocol fulfils several requirements. Firstly yields of RNA extraction directly from environments are usually low (sometimes less than $100 \mathrm{ng} \cdot \mathrm{g}^{-1}$ of soil) and therefore isolation of $\mu \mathrm{g}$ amounts of poly-A mRNA appears, in most cases, almost unfeasible. Secondly, two dimensional electrophoretic separation of cDNAs leads to isolation of sized cDNA pools almost free from contaminations by either longer or shorter cDNAs. Most importantly these pools of amplified cDNAs allow production of large cDNA libraries necessary to capture the gene diversity which characterizes microbial communities. Thirdly, the production of sized cDNA libraries is of direct relevance in the context of functional metatranscriptomics as it should reduce the screening effort for gene categories of defined length which, as a result of size selection, will not be diluted among shorter or longer transcripts. As an example, most glycoside hydrolases (e.g. cellulases, hemicellulases), implicated in organic matter degradation, are encoded by transcripts $1-3 \mathrm{~kb}$ in length and screening for these genes can be limited to the corresponding sized cDNA libraries. In conclusion, we believe that the protocol presented in this paper should facilitate and promote studies of environmental eukaryotic communities, which in a context of environmental biotechnology represent a promising and almost untouched source of genes of interest.

\section{Methods}

RNA extraction from soil

Three soils, two from France (PL and BB) and one from India (UP) were used (Additional file 1). Total RNAs were isolated from soil samples according to Damon et al. [21] for the $\mathrm{BB}$ sample or by using the RNA PowerSoil ${ }^{\circ}$ Total RNA Isolation Kit (Mo Bio laboratories, Carlsbad, CA) for the PL and UP samples. All soil RNA samples were treated with RNase-free DNase I. After a final precipitation step, all the RNAs were dissolved in nuclease-free water. RNA integrity was checked by Bioanalyzer 2100 (Agilent Technologies, USA) electrophoresis and RNA quantity and purity were determined by spectrophotometry (SAFAS UVmc2, SAFAS Monaco).

\section{Synthesis, size fractionation and amplification of CDNAs}

cDNAs were synthesized by using the Mint-2 cDNA synthesis kit (Evrogen, Moscow, Russia) according to the manufacturer's instructions. Briefly, three $\mu \mathrm{g}$ of total soil RNAs were mixed with $10 \mu \mathrm{M}$ of two oligonucleotide adapters. The 3 'end CDS-4M adapter contains (i) an oligo (dT) sequence that anneals to the poly (A) stretch of eukaryotic mRNAs, (ii) a $S f i \mathrm{IB}$ restriction site and (iii) sequence of primer M1. The $5^{\prime}$-end PlugOligo-3M adapter contains (i) an oligo (dG) sequence which anneals to the complementary oligo $(\mathrm{dC})$ stretch added to the 3 '-end of the first-strand cDNA by Mint MMLV RT (ii) a SfiIA restriction site and (iii) sequence of primer M1. The mixture was incubated at $70^{\circ} \mathrm{C}$ for 2 minutes. First-strand cDNAs were synthesized at $42^{\circ} \mathrm{C}$ by Mint $\mathrm{RT}$ in presence of dNTPs, DTT, first-strand buffer and IP-solution in $15 \mu \mathrm{L}$ of total reaction volume. Second-strand cDNA synthesis was carried out by the thermostable Encyclo DNA polymerase (Evrogen) using the M1 primer which 
recognizes both the PlugOligo-3M and CDS-4M adapter sequences. Four $\mu \mathrm{L}$ from first-strand cDNA reaction, i.e. the equivalent of $800 \mathrm{ng}$ of total soil RNA, were used in second-strand cDNA synthesis. Second strand synthesis was followed by a PCR amplification limited to 3 cycles at $95^{\circ} \mathrm{C}$ for $15 \mathrm{sec}, 66^{\circ} \mathrm{C}$ for $20 \mathrm{sec}$ and $72^{\circ} \mathrm{C}$ for $3 \mathrm{~min}$. Resulting double-stranded cDNAs (ds cDNAs) were purified by phenol-chloroform extraction and precipitated.

Size fractionation of cDNAs was performed as described by Wellenreuther et al. [18]. ds cDNAs and a DNA size standard were size fractionated in two separate but identical $0.7 \%$ agarose gels at identical running conditions. After electrophoresis, the gel lanes containing ds cDNAs and DNA size standard were cut out, rotated at $90^{\circ}$ and placed into two separate but identical gel trays. Identical volumes of $1.4 \%$ low melting point agarose (Bioprobe Systems, Montreuil, France) were cast in both the trays and the gels were subjected to electrophoresis at $2.6 \mathrm{~V} . \mathrm{cm}^{-1}$ for $10 \mathrm{~h}$. The unstained gel containing cDNAs was then superimposed over the ethidium bromide stained gel containing the size standard which was visualized using a Dark Reader transilluminator (Clare Chemical Research, Inc., USA). Three gel slices corresponding to the cDNA size fractions A, 0.1-0.5 kb; B, 0.5-1 kb and C, 1-4 kb, were cut out from the unstained gel. cDNAs were extracted from each gel slice by using QIAEX II Gel Extraction Kit (Qiagen, Netherlands), precipitated and amplified by PCR using primer $\mathrm{M} 1$ as described above but using higher number of cycles. Depending on cDNA size fraction and RNA sample, between 22 to 30 cycles were performed for optimal amplification.

\section{Validation of cDNA size fractions}

Validation of the different sized cDNA fractions was performed by running each of them in standard 1\% agarose gels and by PCR amplification, on each of them, of different gene families representative of the different size groups. For fraction $C$, the selected families encoded $\beta$-Tubulin and Elongation factor 1-alpha (EF1 $\alpha)$. They were amplified using respectively primer pairs BTf (GGTAACCAAAT CGGTGCTGCTTTC)/BTr (ACCCTCAGTGTAGTGACC CTTGGC) [22] and EF1f (GTCGTYGTYATYGGHCAY GT)/EF1r (TGYTCNCGRGTYTGNCCRTCYTT) [23]. For fraction $\mathrm{B}$, the selected families encoded 40S Ribosomal protein S3 amplified using primers ribStF (CHSKHACYG ABRTCATCATCCG) and ribStR, (AADCCRTCRGTGAA CTTCATG) (this study) and Peptide methionine sulphoxide reductase amplified using MsrAf (CGCCG CCGGCTGYTTYTGGGG) and MsrAr (ATRGTRGT YNWCATGGACCTGTTCTTGGGGC) [24]. For the smallest fraction A, no conserved gene family in this size range could be identified to design PCR primers that would work on environmental cDNA samples.
Ten ng of each cDNA fraction were used as template in 35 cycles PCR reactions.

\section{cDNA cloning}

cDNA fractions (500 ng) were digested by SfiI which recognizes $S f i \mathrm{IA}$ and $S f i \mathrm{IB}$ sites located in the sequences of PlugOligo-3M and CDS-4M, respectively. Following phenol-chloroform extraction and precipitation, cDNAs were ligated downstream of the $S$. cerevisiae PGK1 promoter in a modified pFL61 yeast expression vector containing SfiIA and SfilB sites [5,25]. Recombinant plasmids were introduced into electro-competent $E$. coli cells (MegaX DH10B $^{\mathrm{TM}} \mathrm{T} 1 \mathrm{R}$ Electrocomp ${ }^{\mathrm{TM}}$ cells, Invitrogen) and at least $10^{6}$ ampicillin resistant bacterial colonies growing on agar medium were collected and pooled to constitute each of the libraries.

\section{Sequencing}

Plasmids were isolated from ten randomly selected bacterial colonies from each of the three libraries constructed from PL sample. cDNA inserts were sequenced from their 5' end and deduced amino acid sequences were used in similarity search (BLASTX) against the GenBank nr eukaryotic protein database (as in December 2013). Resulting cDNA sequences appear in the EMBL database under accession Nos. HG964498 to HG964527.

\section{Additional files}

Additional file 1: Main characteristics of the soil sampling sites.
Additional file 2: cDNA size fractionation from different soils.
Description of data: Agarose gel showing amplified eukaryotic CDNA
fractions A, B and C after size fractionation from (A) Indian soil UP and (B)
French forest soil BB sample. Fraction C of soil BB was cloned in modified
pFL61 vector. (C) The corresponding library (BB-C) was digested with Sfil
enzyme realeasing CDNA inserts of sizes ranging, as expected, between
1 and 4 kb.
Additional file 3: Validation of insert size by colony PCR amplification.
Description of data: CDNA inserts from 42 random colonies from each of the
three libraries PL-A, PL-B and PL-C and 30 random colonies from library BB-C
were amplified by colony PCR. As expected, the sizes of amplified DNA inserts
of each library were confined between their expected size cut offs. Panels A, B
and C are the gel images of separated PCR products from libraries PL-A, PL-B
and PL-C respectively. Panel D is the gel image after migration of PCR
products from library BB-C.
Additional file 4: Protein similarity search of sequenced cDNA
inserts using blastx against GenBank eukaryotic protein sequences.
An e-value threshold of $10^{-5}$ was retained for annotation. aa, amino acids.

Competing interests

The authors declare that they have no competing interests.

\section{Authors' contributions}

RKY, RM and LF designed and coordinated the study. RKY, FB, AZ and MSR carried out the experiments. RKY, RM and LF wrote the manuscript, and PL and MSR reviewed the manuscript. All authors read and approved the final manuscript. 


\section{Acknowledgements}

This study is part of project 4709-1 funded by Indo-French Centre for the Promotion of Advanced Research which granted a post-doctoral fellowship to RKY. We would like to thank Jacques Ranger for access to the Breuil site, and Damien Blaudez and Michel Chalot for access to the Pierrelaye one.

\section{Author details}

${ }^{1}$ Ecologie Microbienne, UMR CNRS 5557, USC INRA 1364, Université Lyon 1, Université de Lyon, Villeurbanne, France. ${ }^{2}$ Department of Biotechnology Thapar University, Patiala 147004, Punjab, India.

Received: 23 April 2014 Accepted: 21 August 2014

Published: 3 September 2014

\section{References}

1. Bailly J, Fraissinet-Tachet L, Verner MC, Debaud JC, Lemaire M, Wesolowski-Louvel M, Marmeisse R: Soil eukaryotic functional diversity, a metatranscriptomic approach. ISME J 2007, 1:632-642.

2. Todaka N, Moriya S, Saita K, Hondo T, Kiuchi I, Takasu H, Ohkuma M, Piero C, Hayashizaki Y, Kudo T: Environmental CDNA analysis of the genes involved in lignocellulose digestion in the symbiotic protist community of Reticulitermes speratus. FEMS Microbiol Ecol 2007, 59:592-599.

3. Kellner $H$, Luis $P$, Portetelle $D$, Vandenbol M: Screening of a soil metatranscriptomic library by functional complementation of Saccharomyces cerevisiae mutants. Microbiol Res 2011, 166:360-368.

4. Damon C, Vallon L, Zimmermann S, Haider MZ, Galeote V, Dequin S, Luis P, Fraissinet-Tachet $L$, Marmeisse R: A novel fungal family of oligopeptide transporters identified by functional metatranscriptomics of soil eukaryotes. ISME J 2011, 5:1871-1880.

5. Lehembre F, Doillon D, David E, Perrotto S, Baude J, Foulon J, Harfouche L, Vallon L, Poulain J, Da Silva C, Wincker P, Oger-Desfeux C, Richaud P, Colpaert V, Chalot M, Fraissinet-Tachet L, Blaudez D, Marmeisse R: Soil metatranscriptomics for mining eukaryotic heavy metal resistance genes. Env Microbio/ 2013, 15:2829-2840

6. Takasaki K, Miura T, Kanno M, Tamaki H, Hanada S, Kamagata Y, Kimura N: Discovery of glycoside hydrolase enzymes in an avicel-adapted forest soil fungal community by a metatranscriptomic approach. PLoS One 2013, 8:e55485.

7. Grant $\mathrm{S}$, Grant WD, Cowan DA, Jones BE, Ma Y, Ventosa A, Heaphy S: Identification of eukaryotic open reading frames in metagenomic CDNA libraries made from environmental samples. Appl Env Microbiol 2006, 72:135-143.

8. Malboeuf CM, Isaacs SJ, Tran NH, Kim B: Thermal effects on reverse transcription: improvement of accuracy and processivity in CDNA synthesis. Biotechniques 2001, 30:1074-1078. 1080, 1082, passim.

9. Theissen H, Etzerodt M, Reuter R, Schneider C, Lottspeich F, Argos P, Luhrmann R, Philipson L: Cloning of the human CDNA for the U1 RNA-associated 70K protein. EMBO J 1986, 5:3209-3217.

10. Edery I, Chu LL, Sonenberg N, Pelletier J: An efficient strategy to isolate full-length cDNAs based on an mRNA cap retention procedure (CAPture). Mol Cell Biol 1995, 15:3363-3371.

11. Carninci P, Kvam C, Kitamura A, Ohsumi T, Okazaki Y, Itoh M, Kamiya M, Shibata K, Sasaki N, Izawa M, Muramatsu M, Hayashizaki Y, Schneider C: High-efficiency full-length CDNA cloning by biotinylated CAP trapper. Genomics 1996, 37:327-336

12. Suzuki Y, Yoshitomo-Nakagawa K, Maruyama K, Suyama A, Sugano S: Construction and characterization of a full length-enriched and a 5'-end-enriched cDNA library. Gene 1997, 200:149-156.

13. Seki M, Narusaka M, Kamiya A, Ishida J, Satou M, Sakurai T, Nakajima M, Enju A, Akiyama K, Oono Y, Muramatsu M, Hayashizaki Y, Kawai J, Carninci P, Itoh M, Ishii Y, Arakawa T, Shibata K, Shinagawa A, Shinozaki K: Functional annotation of a full-length Arabidopsis cDNA collection. Science 2002, 296:141-145.

14. Fernandez C, Gregory WF, Loke P, Maizels RM: Full-length-enriched cDNA libraries from Echinococcus granulosus contain separate populations of oligo-capped and trans-spliced transcripts and a high level of predicted signal peptide sequences. Mol Biochem Parasitol 2002, 122:171-180

15. Seki N, Ohira M, Nagase T, Ishikawa K, Miyajima N, Nakajima D, Nomura N, Ohara O: Characterization of CDNA clones in size-fractionated cDNA libraries from human brain. DNA Res 1997, 4:345-349.
16. Draper MP, August PR, Connolly T, Packard B, Call KM: Efficient cloning of full-length cDNAs based on CDNA size fractionation. Genomics 2002, 79:603-607.

17. Zhu YY, Machleder EM, Chenchik A, Li R, Siebert PD: Reverse transcriptase template switching: a SMART approach for full-length CDNA library construction. Biotechniques 2001, 30:892-897.

18. Wellenreuther R, Schupp I, Poustka A, Wiemann S: SMART amplification combined with CDNA size fractionation in order to obtain large full-length clones. BMC Genomics 2004, 5:36.

19. Urich T, Lanzen A, Qi J, Huson DH, Schleper C, Schuster SC: Simultaneous assessment of soil microbial community structure and function through analysis of the meta-transcriptome. PLoS One 2008, 3:e2527.

20. Daniel R: The metagenomics of soil. Nature Rev Microbiol 2005, 3:470-478.

21. Damon C, Barroso G, Ferandon C, Ranger J, Fraissinet-Tachet L, Marmeisse R: Performance of the COX1 gene as a marker for the study of metabolically active Pezizomycotina and Agaricomycetes fungal communities from the analysis of soil RNA. FEMS Microbiol Ecol 2010, 74:693-705.

22. Glass NL, Donaldson GC: Development of primer sets designed for use with the PCR to amplify conserved genes from filamentous ascomycetes. Appl Env Microbiol 1995, 61:1323-1330.

23. Rehner SA, Buckley E: A Beauveria phylogeny inferred from nuclear ITS and EF1-alpha sequences: evidence for cryptic diversification and links to Cordyceps teleomorphs. Mycologia 2005, 97:84-98.

24. Lewis CT, Bilkhu S, Vincent R, Eberhardt U, Szoke S, Seifert KA, Lévesque CA: Identification of fungal DNA barcode targets and PCR primers based on Pfam protein families and taxonomic hierarchy. Open Appl Inform J 2011, 5:30-44.

25. Minet M, Dufour ME, Lacroute F: Complementation of Saccharomyces cerevisiae auxotrophic mutants by Arabidopsis thaliana cDNAs. Plant J 1992, 2:S-422.

doi:10.1186/1472-6750-14-80

Cite this article as: Yadav et al:: Construction of sized eukaryotic CDNA libraries using low input of total environmental metatranscriptomic RNA. BMC Biotechnology 2014 14:80.

\section{Submit your next manuscript to BioMed Central and take full advantage of:}

- Convenient online submission

- Thorough peer review

- No space constraints or color figure charges

- Immediate publication on acceptance

- Inclusion in PubMed, CAS, Scopus and Google Scholar

- Research which is freely available for redistribution

Submit your manuscript at www.biomedcentral.com/submit
C Biomed Central 